\title{
Conduta de enfermagem em gestantes cardiopatas contaminadas pela Covid-19
}

\author{
Nursing conduct in pregnant women with heart disease contaminated by Covid-19 \\ Conducta de lactancia en mujeres embarazadas con enfermedad cardíaca contaminada por Covid-
}

Daniele Vignoli Ribeiro

ORCID: https://orcid.org/0000-0001-5296-8302 Associação Brasileira de Enfermeiros Cientistas, Brasil

E-mail: vignoliribeiro@gmail.com

Priscila Gramata da Silva Vitorino

ORCID: https://orcid.org/0000-0002-1201-6945 Associação Brasileira de Enfermeiros Cientistas, Brasil E-mail: prigramaenf@yahoo.com.br

Judith Victoria Castillo Mejía

ORCID: https://orcid.org/0000-0001-5257-6878 Associação Brasileira de Enfermeiros Cientistas, Brasil E-mail: iker07castillo@live.com

Luana de Oliveira Hernandes

ORCID: https://orcid.org/0000-0001-8828-7146 Associação Brasileira de Enfermeiros Cientistas, Brasil E-mail: luanaoliveira2306@gmail.com

Thais Oliveira de Paula Lima

ORCID: https://orcid.org/0000-0002-0706-1098 Associação Brasileira de Enfermeiros Cientistas, Brasil E-mail: thaisoplima@gmail.com

Natasha Vila Chã

ORCID: https://orcid.org/0000-0002-9781-454X Associação Brasileira de Enfermeiros Cientistas, Brasil E-mail: natashavilacha@hotmail.com

Victor Hugo de Paula Flauzino

ORCID: https://orcid.org/0000-0001-5156-0030 Associação Brasileira de Enfermeiros Cientistas, Brasil

E-mail: prof.victorflauzino@unyleya.edu.br

Thays Vieira Cusato

ORCID: https://orcid.org/0000-0002-3766-6530 Associação Brasileira de Enfermeiros Cientistas, Brasil E-mail: thays.vieira.cusato@gmail.com

Daiana Moreira Gomes

ORCID: https://orcid.org/0000-0001-9387-0619 Associação Brasileira de Enfermeiros Cientistas, Brasil E-mail: daigomes_87@hotmail.com

Jonas Magno dos Santos Cesário

ORCID: https://orcid.org/0000-0003-1785-3555 Associação Brasileira de Enfermeiros Cientistas, Brasil E-mail: prof.jonasmagno@unyleya.edu.br

\begin{abstract}
Resumo
As condições clínicas de uma mulher antes da gestação são bem diferentes daquelas com uma com gestação em curso. As alterações fisiológicas apresentadas durante a gestação e que conferem à mulher vulnerabilidade foram consideradas como de risco elevado, quando associadas ao covid-19. O objetivo principal deste estudo foi identificar os principais cuidados de enfermagem realizados em gestantes cardiopatas contaminadas pela covid-19 e, mais especificamente, descrever os principais diagnósticos de enfermagem nas gestantes cardiopatas contaminadas pela covid-19 e identificar as principais intervenções de enfermagem em gestantes cardiopatas contaminadas pela covid19. Trata-se de uma pesquisa descritiva, com busca de artigos realizada em bibliotecas digitais. Observou-se que, apesar das pesquisas mostrarem que as gestantes possuem um maior risco para a infeção por covid-19, alguns autores observaram que as consequências para o feto e recém-nascido também podem ser graves, principalmente se a infecção ocorrer no último trimestre da gestação. Faz-se imprescindível que o cuidado humanizado e individualizado se mantenha presente, pois as gestantes fazem parte de um grupo de pacientes que precisam de uma atenção crítica por conta de suas alterações fisiológicas e imunológicas.
\end{abstract}

Palavras-chave: Infecções por coronavírus; Gestação; Cardiopatia. 


\begin{abstract}
The clinical conditions of a woman before pregnancy are quite different from those with an ongoing pregnancy. Physiological changes presented during pregnancy and that make women vulnerable were at high risk when associated with covid-10. The main objective of this study was to identify the main nursing care provided to pregnant women with heart disease contaminated by covid-19 and, more specifically, to describe the main nursing diagnoses in pregnant women with heart disease infected by covid-19 and to identify the main nursing interventions in pregnant women with heart disease contaminated by covid-19. This is a descriptive research, with a search for articles carried out in digital libraries. It was observed that, despite research showing that pregnant women are at greater risk for infection by covid-19, some authors noted that the consequences for the fetus and newborn can also be serious, especially if the infection occurs in the last trimester. of pregnancy. It is essential that humanized and individualized care remains present, as pregnant women are part of a group of patients who need critical attention due to their physiological and immunological changes.
\end{abstract}

Keywords: Coronavirus infections; Pregnancy; Cardiopathy.

\title{
Resumen
}

Las condiciones clínicas de una mujer antes del embarazo son bastante diferentes a las de un embarazo en curso. Los cambios fisiológicos que se presentan durante el embarazo y que hacen que las mujeres sean vulnerables se consideraron de alto riesgo cuando se asociaron con el covid-10. El objetivo principal de este estudio fue identificar los principales cuidados de enfermería brindados a las gestantes con cardiopatía contaminada por covid-19 y, más específicamente, describir los principales diagnósticos de enfermería en gestantes con cardiopatía infectada por covid19 e identificar las principales intervenciones de enfermería en gestantes con cardiopatías contaminadas por covid-19. Se trata de una investigación descriptiva, con búsqueda de artículos realizada en bibliotecas digitales. Se observó que, a pesar de que las investigaciones muestran que las mujeres embarazadas tienen un mayor riesgo de infección por covid-19, algunos autores señalaron que las consecuencias para el feto y el recién nacido también pueden ser graves, especialmente si la infección ocurre en el último trimestre del embarazo. Es fundamental que la atención humanizada e individualizada permanezca presente, ya que las mujeres embarazadas forman parte de un grupo de pacientes que necesitan atención crítica por sus cambios fisiológicos e inmunológicos.

Palabras clave: Infecciones por coronavirus; Embarazo; Enfermedad cardíaca.

\section{Introdução}

A gestação é um período diferenciado na vida da mulher, pois todo o organismo se adapta à nova realidade de concepção de uma nova vida. Para isso, todos os sistemas orgânicos do corpo sofrem alterações no seu funcionamento para atender às necessidades da gestante e do feto que está em desenvolvimento. Um dos sistemas que sofre alterações significativas é o sistema cardiocirculatório, como a hipervolemia (com consequente anemia fisiológica), o aumento do débito cardíaco (para garantir o suprimento sanguíneo para gestante e feto), a diminuição da resistência vascular periférica, que está diretamente ligada à alta concentração de progesterona (progesterona causa hipotonia nos vasos sanguíneos e dificulta o retorno do sangue venoso) e diminuição da pressão arterial média (Flauzino et al., 2021).

As condições clínicas de uma mulher antes da gestação são bem diferentes daquelas com uma com gestação em curso. As alterações fisiológicas apresentadas durante a gestação e que conferem à mulher vulnerabilidade foram consideradas como de risco elevado, quando associadas ao coronavírus. A covid-19 foi descrita como uma doença que afeta o sistema respiratório e, posteriormente, a circulação sistêmica, evoluiu para uma pandemia após os milhares de casos espalhados confirmados pelo mundo todo. Caracterizou-se por sintomas clássicos, tais como febre, tosse, dispneia, cansaço, e outros sintomas, alguns indivíduos eram assintomáticos e não apresentaram nenhum sintoma da covid-19 (Cesário et al.,2021).

De acordo com Holanda et al. (2020), as pessoas que sofrem com problemas cardiovasculares representam cerca de $30 \%$ das mortes, e a estimativa é que até 2040 essa porcentagem chegue a 250\%. Com o advento da covid-19, esse risco de eventos cardiovasculares aumentou consideravelmente, e as gestantes foram inseridas no grupo de risco por naturalmente apresentarem alterações cardiovasculares pertinentes à adaptação do organismo à gestação. Apesar de existirem poucos estudos disponíveis que correlacionem as gestantes cardiopatas à covid-19, observou-se que 22\% das gestantes sintomáticas necessitaram de cuidados intensivos sob o risco de morte materna e fetal. Estudos realizados pela UW School of Medicine (2021) mostraram que, além do aumento do risco de morte, as mulheres também apresentavam maior probabilidade de ter 
parto prematuro, pré-eclâmpsia, que exigem internação na Unidade de Terapia Intensiva (UTI) e/ou intubação.

Nascimento, Cardoso e Neves (2020) afirmaram que as principais afecções cardíacas potencializadas pela covid-19 foram arritmias, choque cardiogênico, isquemia miocárdica e miocardite. Evidências científicas apontaram que a covid-19 interage com o sistema cardiovascular e pode ocasionar lesão no músculo cardíaco. Essas lesões corresponderam a 23\% dos casos de internação de pacientes cardiopatas com covid-19 em UTIs (Pereira \& Correa, 2020).

A justificativa para a realização deste estudo foi fundamentada em dois pontos importantes: o primeiro, foram identificados em 40,4\% dos pacientes complicações cardiovasculares após contraírem a covid-19 (Matos et al., 2021); e o segundo ponto baseou-se no fato de que as cardiopatias representaram o maior número de casos de óbito materno de forma indireta no ciclo gravidez-puerpério. Além disso, a escassez de estudos que correlacionassem os diagnósticos de enfermagem e os cuidados para a gestante foram outro fator que motivou a elaboração deste estudo.

Devido ao elevado índice de contaminação e risco aumentado de complicações pela covid em pacientes com comorbidades, gestantes cardiopatas foram categorizadas como parte do grupo de risco, o que demandou maior atenção por parte dos profissionais e modificou toda a rotina da equipe de enfermagem das unidades obstétricas. A doença cardíaca e o fato de a gestação alterar o sistema imunológico podem contribuir para uma maior propensão ao desenvolvimento de quadros mais graves de infecção pela covid-19. O presente estudo procurou responder a seguinte pergunta: qual deve ser a conduta de enfermagem em gestantes cardiopatas contaminadas pela covid-19?

Desta forma, foi traçado como objetivo principal, identificar os principais cuidados de enfermagem realizados em gestantes cardiopatas contaminadas pela covid-19 e, mais especificamente, descrever os principais diagnósticos de enfermagem nas gestantes cardiopatas contaminadas pela covid-19 e identificar as principais intervenções de enfermagem em gestantes cardiopatas contaminadas pela covid-19.

\section{Metodologia}

Este estudo é uma pesquisa descritiva, que de acordo com Cesário, Flauzino e Mejia (2020) é direcionada para responder alguma dúvida ou questionamento, que pode incluir artigos científicos publicados em periódicos acadêmicos. Dessa forma, inicialmente buscou-se reunir evidências para responder ao questionamento de pesquisa. Na etapa inicial, a busca foi realizada em bibliotecas digitais, que permitem uma busca em bases de dados próprias por meio da utilização de descritores padronizados. No DeCS (Descritores em Ciências da Saúde), foram encontrados os seguintes descritores: infecções por coronavírus, gestação e cardiopatia.

Os bancos de dados utilizados foram o Google Acadêmico, BVS (biblioteca virtual em saúde) e SciELO (Scientific Electronic Library Online). No Google Acadêmico utilizou-se cada um dos descritores entre aspas ("')). Na BVS, foi utilizado a opção pesquisa avançada, selecionadas as bases da BDENF (Banco de Dados em Enfermagem), LILACS (Literatura LatinoAmericana e do Caribe em Ciências da Saúde) e o operador lógico booleano "OR" e "AND" e por último na SciELO, foi utilizada a opção pesquisa avançada e o operador lógico booleano "OR" e "AND".

Estabeleceu-se como critérios de inclusão: artigos acadêmicos publicados entre 2020 e 2021, na língua portuguesa, disponíveis de forma gratuita e nos bancos de dados já mencionados, que respondessem à pergunta de pesquisa. Excluíram-se artigos repetidos encontrados nas bases de dados, resumos, artigos inferiores a 2020 e artigos que não respondiam o problema da pesquisa. A coleta dos dados foi realizada no mês de março, por dois pesquisadores de forma independente. Os resultados das buscas pelos dados e do número final de publicações que irão compor a revisão são apresentados conforme orientações do Prisma na forma de fluxograma. (Peters, 2015), conforme a Figura 1: 
Figura 1. Fluxograma de Prisma.

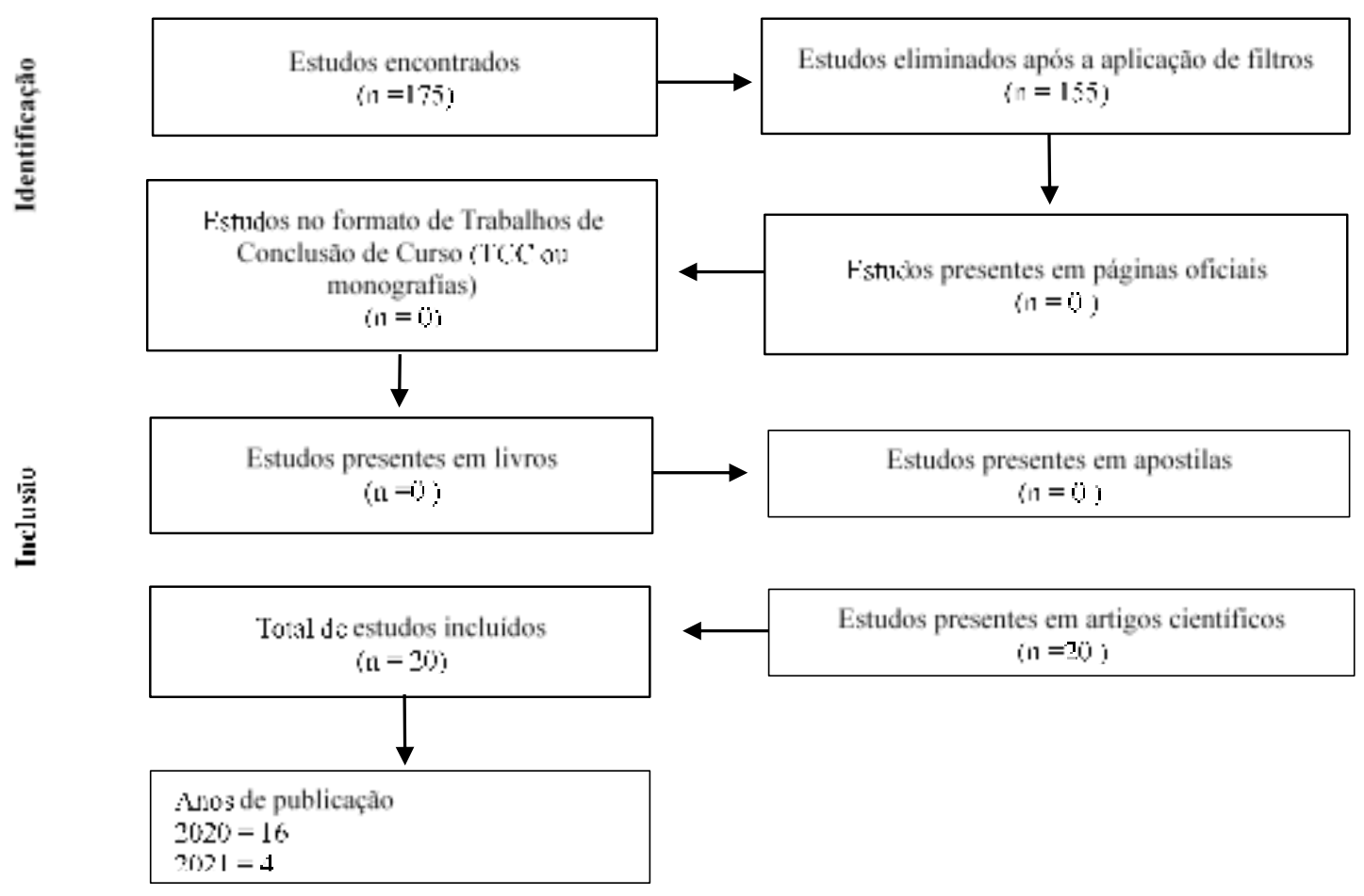

Fonte: Autores (2021).

Inicialmente foram encontrados 125 estudos e após a aplicação dos filtros, conforme os critérios de inclusão e exclusão, foram eliminados 104 estudos. Não foram considerados estudos em páginas oficiais da internet, monografias, apostilas e livros. Ao final foram incluídos 20 artigos científicos.

\section{Resultados}

A Tabela 1 é composta das variáveis de inclusão e exclusão, que mostra a distribuição inicial dos artigos científicos encontrados nas bases de dados da BVS, ScIELO e Google Acadêmico. É possível notar que o maior aproveitamento de periódicos foi na base da BVS junto com o Google Acadêmico e por último a ScIELO, o resultado apresentou uma amostra final de 20 artigos científicos.

Tabela 1. Artigos inclusos neste estudo.

\section{BVS ScIELO Google Acadêmico Amostra final}

$\begin{array}{lclclcl}\text { Total } & 75 & \text { Total } & 20 & \text { Total } & 80 & \\ \text { Excluídos } & 67 & \text { Excluídos } & 16 & \text { Excluídos } & 72 & 20 \text { artigos } \\ \text { Incluídos } & 8 & \text { Incluídos } & 4 & \text { Incluídos } & 8 & \end{array}$

Fonte: Autores (2021).

Durante a realização da pesquisa, os resultados encontrados foram divididos em duas categorias, A e B, de acordo com a sua temática. A categoria A é composta pelos principais diagnósticos de enfermagem nas gestantes cardiopatas contaminadas pela covid-19 e na categoria B as principais intervenções de enfermagem em gestantes cardiopatas contaminadas 
pela covid-19. No Quadro 1, os artigos de revisão da literatura encontrados e utilizados na Categoria A contém as seguintes variáveis: autor, título, objetivo e periódico.

Quadro 1. Artigos incluídos na categoria temática A.

\begin{tabular}{|c|c|c|c|}
\hline Autor/ano & Título & Objetivos & Tipo de estudo \\
\hline Ávila \& Carvalho, 2020 & $\begin{array}{l}\text { Covid-19: um novo desafio para a } \\
\text { cardiopatia na gravidez }\end{array}$ & $\begin{array}{l}\text { Dissertar sobre os aspectos } \\
\text { fisiopatológicos da covid-19 } \\
\text { associados à gestante cardiopata }\end{array}$ & Revisão sistemática \\
\hline $\begin{array}{l}\text { Almeida, Portugal \& Assis, } \\
2020\end{array}$ & $\begin{array}{l}\text { Gestantes e covid-19: isolamento } \\
\text { como fator de impacto físico e } \\
\text { psíquico. }\end{array}$ & $\begin{array}{l}\text { Refletir sobre os impactos } \\
\text { inerentes do isolamento social } \\
\text { proveniente da pandemia do novo } \\
\text { coronavírus (covid-19) na saúde } \\
\text { das gestantes }\end{array}$ & Revisão de literatura \\
\hline Feitosa et al., 2020 & Covid-19 e o coração. & $\begin{array}{lccr}\text { Refletir } & \text { sobre } & \text { os } & \text { impactos } \\
\text { causados pela covid-19 em } & \text { em } \\
\text { pacientes } & \text { com afecções } \\
\text { cardiovasculares }\end{array}$ & Pesquisa qualitativa \\
\hline Estrela et al., 2020 & $\begin{array}{l}\text { Gestantes no contexto da } \\
\text { pandemia da covid-19: reflexões } \\
\text { e desafios }\end{array}$ & $\begin{array}{l}\text { Refletir sobre o estar gestante em } \\
\text { tempos de pandemia da covid-19 }\end{array}$ & Revisão integrativa \\
\hline Ribeiro et al., 2021 & $\begin{array}{l}\text { Repercussões da covid-19 para as } \\
\text { gestantes. }\end{array}$ & $\begin{array}{l}\text { Refletir acerca das repercussões } \\
\text { da covid-19 para as gestantes. }\end{array}$ & Revisão integrativa \\
\hline Melo \& Cruz, 2020 & $\begin{array}{l}\text { Gestação no cárcere: garantia aos } \\
\text { direitos reprodutivos em tempos } \\
\text { de pandemia da covid-19 }\end{array}$ & $\begin{array}{l}\text { Identificar as principais violações } \\
\text { e desafios para a efetivação dos } \\
\text { direitos reprodutivos das } \\
\text { mulheres em sistema prisional, } \\
\text { sobretudo em contexto de } \\
\text { pandemia pela covid-19 }\end{array}$ & Revisão bibliográfica \\
\hline Santos et al., 2020 & $\begin{array}{l}\text { Posicionamento sobre covid-19 e } \\
\text { gravidez em mulheres cardiopatas } \\
\text { - Departamento de Cardiologia } \\
\text { da Mulher da Sociedade } \\
\text { Brasileira de Cardiologia - } 2020\end{array}$ & $\begin{array}{l}\text { Relatar aspectos da covid-19 } \\
\text { durante a gravidez e sua } \\
\text { concomitância com cardiopatias, } \\
\text { além de propor recomendações } \\
\text { que possam contribuir para } \\
\text { protocolos de assistência a } \\
\text { gestantes cardiopatas durante a } \\
\text { presente pandemia. }\end{array}$ & Revisão integrativa \\
\hline $\begin{array}{l}\text { Nascimento, Cardoso \& } \\
\text { Neves, } 2020\end{array}$ & $\begin{array}{l}\text { Principais desfechos fatais em } \\
\text { indivíduos } \\
\text { acometidos por covid-19 }\end{array}$ & $\begin{array}{l}\text { Identificar os principais desfechos } \\
\text { fatais em indivíduos cardiopatas } \\
\text { acometidos por covid-19 }\end{array}$ & Revisão integrativa \\
\hline Guedes et al., 2020 & $\begin{array}{l}\text { Aspectos gerais da covid-19 na } \\
\text { saúde de gestantes e recém- } \\
\text { nascidos: uma breve revisão }\end{array}$ & $\begin{array}{l}\text { Realizar uma revisão integrativa } \\
\text { da literatura contendo as } \\
\text { principais informações acerca do } \\
\text { impacto da covid- } 19 \text { na saúde dos } \\
\text { recém-nascidos e gestantes }\end{array}$ & $\begin{array}{l}\text { Revisão narrativa qualitativa } \\
\text { da literatura científica }\end{array}$ \\
\hline Rodrigues et al., 2020 & $\begin{array}{llll}\text { Covid-19: } & \text { sistema } & \text { renal } & \text { e } \\
\text { cardíaco } & & & \end{array}$ & $\begin{array}{l}\text { Descrever a vulnerabilidade de } \\
\text { pacientes portadores de doenças } \\
\text { renais e cardíacas à severidade da } \\
\text { infecção por SARS-CoV-2 }\end{array}$ & Revisão bibliográfica \\
\hline
\end{tabular}

Fonte: Autores (2021).

No Quadro 2, são apresentados os artigos de revisão da literatura encontrados e utilizados na categoria B, que contêm as seguintes variáveis: autor, título, objetivo e periódico. O quadro B possui um total de 10 periódicos que contribuíram na comparação dos assuntos abordados na temática B. 
Quadro 2. Artigos incluídos na categoria temática B.

\begin{tabular}{|c|c|c|c|}
\hline Autor/ano & Título & Objetivos & Tipo de estudo \\
\hline $\begin{array}{l}\text { Marquard, Bertoldi \& } \\
\text { Carvalho, } 2020\end{array}$ & $\begin{array}{l}\text { Assistência de enfermagem a } \\
\text { gestantes atendidas nos serviços } \\
\text { de saúde em tempos de } \\
\text { pandemia: covid-19 }\end{array}$ & $\begin{array}{l}\text { Descrever a assistência de } \\
\text { enfermagem a gestantes usuárias } \\
\text { dos serviços de saúde em meio à } \\
\text { infeção pelo novo coronavírus, as } \\
\text { características clínicas, além dos } \\
\text { desfechos maternos e neonatais. }\end{array}$ & $\begin{array}{l}\text { Revisão narrativa } \quad \text { da } \\
\text { literatura }\end{array}$ \\
\hline $\begin{array}{l}\text { Alburquerque, Monte } \quad \& \\
\text { Araújo, } 2020\end{array}$ & $\begin{array}{l}\text { Implicações da covid-19 para } \\
\text { pacientes gestantes }\end{array}$ & $\begin{array}{l}\text { Manejo e medidas de controle da } \\
\text { covid-19 para as gestantes e os } \\
\text { possíveis riscos durante } \\
\text { transmissão vertical e } \\
\text { amamentação. }\end{array}$ & Revisão bibliográfica \\
\hline Holanda et al., 2020 & $\begin{array}{l}\text { Infecção por covid-19 em } \\
\text { gestante cardiopata }\end{array}$ & $\begin{array}{l}\text { Discutir a necessidade de suporte } \\
\text { avançado de vida para as gestantes } \\
\text { com cardiopatias }\end{array}$ & Pesquisa qualitativa \\
\hline Teixeira et al., 2021 & 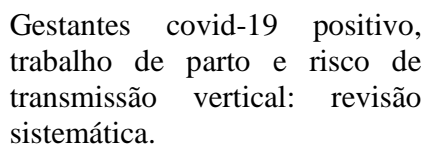 & $\begin{array}{l}\text { Observar os manejos com gestantes } \\
\text { covid-19 positivo, o risco da } \\
\text { transmissão vertical e os cuidados } \\
\text { durante o trabalho de parto. }\end{array}$ & Revisão sistemática \\
\hline Cardoso et al., 2020 & $\begin{array}{l}\text { Covid-19 na gestação: uma } \\
\text { revisão integrativa }\end{array}$ & $\begin{array}{l}\text { Identificar, sintetizar e analisar as } \\
\text { literaturas referentes ao cuidado } \\
\text { com covid-19 na gestação }\end{array}$ & Revisão integrativa \\
\hline Lima et al., 2021 & $\begin{array}{l}\text { Estratégias de educação em } \\
\text { saúde às gestantes e puérperas } \\
\text { no enfrentamento à pandemia de } \\
\text { covid- } 19 .\end{array}$ & $\begin{array}{l}\text { Elencar as estratégias de educação } \\
\text { em saúde voltadas às gestantes e } \\
\text { puérperas no contexto da pandemia } \\
\text { pela covid- } 19 \text {. }\end{array}$ & Revisão integrativa \\
\hline Jesus et al., 2020 & $\begin{array}{l}\text { Gestante com covid-19 } \\
\text { submetida à cesariana por } \\
\text { sofrimento fetal: primeiro relato } \\
\text { de caso descrito no Brasil }\end{array}$ & 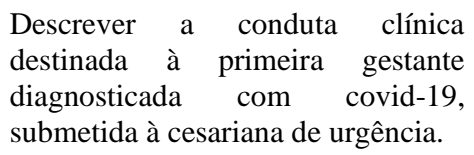 & Relato de caso \\
\hline Pereira \& Correa, 2020 & $\begin{array}{l}\text { Uso da tele consulta pelo } \\
\text { enfermeiro a cardiopatas: uma } \\
\text { reflexão durante pandemia por } \\
\text { covid-19 no Brasil }\end{array}$ & $\begin{array}{l}\text { Refletir sobre o potencial risco do } \\
\text { cardiopata e a atuação do } \\
\text { enfermeiro por meio de orientações } \\
\text { em saúde na tele consulta }\end{array}$ & Estudo de reflexão \\
\hline Rondelli et al., 2020 & $\begin{array}{l}\text { Assistência às gestantes e } \\
\text { recém-nascidos no contexto da } \\
\text { infecção covid-19: uma revisão } \\
\text { sistemática }\end{array}$ & $\begin{array}{l}\text { Explorar e sintetizar as evidências } \\
\text { disponíveis na literatura científica } \\
\text { voltadas para a atenção e o manejo } \\
\text { de gestantes e recém-nascidos } \\
\text { suspeitos ou infectados pelo vírus } \\
\text { SARS-CoV-2 }\end{array}$ & Revisão sistemática \\
\hline Cesário et al., 2021 & $\begin{array}{l}\text { Cuidados de enfermagem para o } \\
\text { paciente com infarto agudo do } \\
\text { miocárdio portador de covid- } 19 \text {. }\end{array}$ & $\begin{array}{l}\text { Descrever os cuidados de } \\
\text { enfermagem para o paciente com } \\
\text { infarto agudo do miocárdio portador } \\
\text { de covid- } 19 \text {. }\end{array}$ & $\begin{array}{l}\text { Revisão bibliográfica } \\
\text { abordagem descritiva } \\
\text { qualitativa }\end{array}$ \\
\hline
\end{tabular}

Fonte: Autores (2021).

\section{Discussão}

\section{Categoria A - Principais diagnósticos de enfermagem nas gestantes cardiopatas contaminadas pela covid-19}

Após o início da pandemia pelo coronavírus, o isolamento social foi implementado por diversos governos estaduais para minimizar a disseminação do vírus. Devido às alterações imuno e fisiológicas decorrentes da gravidez, as gestantes foram orientadas a manter o cuidado e a precaução para minimizar o risco de contaminação devido ao elevado risco de contágio ocasionado pelo vírus (Almeida, Portugal \& Assis, 2020).

As alterações fisiológicas e hormonais apresentadas pela gestante tornaram-na vulnerável à contaminação pela covid19 e o quadro clínico observado variou entre sintomas de baixa intensidade, tais como fadiga, dispneia, diarreia, congestão 
nasal e coriza ou complicações mais graves, como a síndrome respiratória aguda grave. O impacto emocional na saúde mental durante o período gestacional e pandemia foi evidenciado por ansiedade, ataques de pânico, culpa, insônia, perda de apetite e falta de concentração (Estrela et al., 2020). Devido à carência de estudos relacionando gestantes e covid-19, poucas informações a respeito da sintomatologia foram delimitadas. Entretanto, foi observado que as alterações fisiológicas apresentadas durante o período gestacional mascararam os sinais e sintomas comuns encontrados na covid-19, o que resultou em diagnóstico tardio da doença nas gestantes (Cardoso et al., 2020).

Os sinais e sintomas respiratórios encontrados na gestante acometida pela covid-19 tiveram rápida progressão, em contrapartida foram identificados também casos de pacientes assintomáticas. Alguns diagnósticos que explicitaram os riscos para a gestante foram hipóxia materna, choque séptico e alterações fisiológicas com consequente sofrimento fetal. Um dado importante mencionado pelo autor é que a contaminação pelo vírus não ocasionou transmissão vertical da doença (Teixeira $e t$ al., 2021).

O período de reclusão domiciliar foi caracterizado por intensas alterações ligadas aos aspectos físico e emocional. Desequilíbrio fisiológico, déficit de volume de líquidos, ansiedade, relacionamento interpessoal familiar alterado, comunicação prejudicada, mobilidade física prejudicada, déficit de autocuidado, dor, retenção urinária, amamentação ineficaz e risco de tromboembolismo venoso foram alterações identificadas pelo autor desde a gestação até o pós-parto (Almeida, Portugal \& Assis, 2020). As alterações clínicas identificadas em gestantes contaminadas pelo coronavírus foram disfunção metabólica e alterações na coagulação. As gestantes com comorbidades cardíacas associadas à contaminação pelo coronavírus apresentaram risco elevado de interrupção abrupta da gestação, parto prematuro, presença do vírus no líquido amniótico e no sangue do cordão umbilical (Albuquerque, Monte \& Araújo. 2020).

Santos et al. (2020), destacam que gestantes cardiopatas apresentaram maior risco de complicações cardíacas graves, o que demandou da equipe de enfermagem maior atenção às alterações hemodinâmicas apresentadas por essas pacientes. Os principais diagnósticos de enfermagem encontrados na paciente gestante cardiopata com covid-19 foram proteção ineficaz, risco de desequilíbrio eletrolítico, volume de líquidos excessivo relacionado a retenção hídrica, diarreia, troca de gases prejudicada, débito cardíaco diminuído, fadiga, padrão respiratório ineficaz, ventilação espontânea prejudicada, sofrimento espiritual, hipertermia, dor aguda e náusea.

Feitosa $e t$ al. (2020) ratificaram que as gestantes com cardiopatia ou hipertensão arterial e infecção pelo sars-cov-2 foram consideradas pacientes de alto risco para mortalidade em decorrência das alterações fisiopatológicas apresentadas, o que incluiu sintomas característicos da doença cardíaca e contaminação pela covid-19. Concluiu-se que a triagem deveria ser diferenciada com o objetivo de direcionar as estratégias na assistência, no tratamento e na prevenção de complicações durante a gravidez, a fim de contribuir para a redução dos efeitos adversos.

Rondelli et al. (2020) mencionaram que a maioria dos bebês de mães contaminadas não apresentou nenhum processo infeccioso. Sugeriu-se que os casos positivos tenham ocorrido após o parto por meio do contato com a equipe e/ou procedimentos. Apesar da baixa incidência da covid-19 em recém-nascidos, a infecção materna trouxe consequências graves para o bem-estar fetal e neonatal em alguns casos.

\section{Categoria B - As principais intervenções de enfermagem gestantes cardiopatas contaminadas pela covid-19.}

Diante do cenário imposto pela pandemia, os profissionais de saúde que atuam em unidades obstétricas, serviço essencial dentro de um hospital, passaram por treinamentos e atualizações para garantir uma atuação segura durante a pandemia. Nos serviços de atenção básica, o atendimento pré-natal não foi suspenso, mas houveram algumas adaptações. Em caso de suspeita ou confirmação da doença, para gestações de baixo risco, as consultas foram adiadas por até 14 dias, com contato não presencial para caso de dúvidas. Em gestações de alto risco este acompanhamento também não foi interrompido, 
objetivou-se aumento na vigilância fetal e avaliação do crescimento uterino. Nestes casos, as consultas foram realizadas por meio da telemedicina ou atendimentos presenciais nos casos de maior necessidade. As gestantes foram orientadas a usar máscara cirúrgica durante todo o período do atendimento presencial, bem como utilizar via de entrada separada (Rondelli et al., 2020).

Durante o trabalho de parto, foi necessário manter vigilância fetal e aferir a temperatura, frequência respiratória e oximetria materna a cada hora e manter os níveis de saturação de oxigênio acima de $94 \%$. Em relação à via de parto, não foram encontrados estudos conclusivos. Desta forma, ficou a critério da gestante ou indicação obstétrica. Houve controvérsias acerca do alojamento conjunto. Alguns autores não recomendaram o contato devido ao risco de transmissão da mãe para o recémnascido. Já outras fontes defenderam a prática, com adoção de medidas de precaução. Em ambos os casos, foram avaliados os riscos e benefícios dessa separação (Rondelli et al., 2020).

Em relação à amamentação, ainda não foram publicados estudos conclusivos sobre a contaminação pelo leite materno. No entanto, foi observada probabilidade reduzida de uma possível transmissão vertical por essa via. Se a mãe optasse pela oferta de leite através do seio, recomenda-se o uso da máscara durante todo o período e a higienização das mãos antes da alimentação. No momento da ordenha do leite materno, as pacientes foram orientadas a utilizar a máscara, higienizar as mãos antes do procedimento, bem como a higienizar todas as peças que entraram em contato com o leite materno (Guedes et al., 2020). Para que o cuidado às pacientes cardiopatas fosse eficaz, foi necessária a implantação da sistematização dos serviços de enfermagem específica para esta situação especial, com um plano de cuidados individualizado, diagnósticos de enfermagem e medidas de intervenção que atendessem às necessidades das pacientes, bem como a inclusão de suporte psicológico, tanto às pacientes quanto à família (Cesário et al., 2021).

A equipe de enfermagem realizou consultas de acompanhamento, esclareceu dúvidas, orientou sobre as formas de precauções da contaminação juntamente com a equipe multidisciplinar, realizou assistência direcionada com o objetivo de controlar os efeitos adversos da contaminação e patologias associadas (Feitosa et al., 2020). O cuidado às pacientes que necessitaram de internação seguiu criteriosamente os protocolos de precaução já existentes. $\mathrm{O}$ uso dos equipamentos de proteção individual (EPI) foi de extrema importância, assim como a higienização das mãos, o cuidado redobrado ao manejo das pacientes transferidas e quarto de isolamento com pressão negativa. Foi observada a importância do monitoramento dos batimentos cardiofetais na avaliação da vitalidade fetal, independentemente da idade gestacional. Com relação à cesariana, casos suspeitos ou confirmados puderam usar a mesma sala cirúrgica, desde que o caso suspeito fosse o primeiro (Cardoso et al., 2020). A equipe de enfermagem realizou um planejamento para assistir à gestante e minimizar os danos ocasionados durante a gestação concomitantes com a contaminação pelo coronavírus. Após avaliação destes fatores, a detecção precoce e a prevenção foram primordiais para minimizar as complicações obstétricas e adversas (Albuquerque, Monte \& Araújo, 2020).

As medidas tomadas com a finalidade de minimizar impactos físicos e emocionais nas gestantes constituíram-se de consultas de orientação e sinalização de dúvidas via o sistema de telemedicina, as consultas presenciais de pré-natal foram reduzidas para as gestantes de baixo risco, a fim de preservar a integridade física da gestante e do feto. Aspectos emocionais foram abordados com psicoterapia realizada de forma online (Ribeiro et al., 2021).

Após o Conselho Federal de Enfermagem (COFEN) autorizar o enfermeiro a realizar teleconsultas com intuito de fornecer informações para a população em geral sobre a covid-19, notou-se o aumento da disseminação de orientações importantes quanto à forma de contaminação, importância de manter o distanciamento, e principalmente refletir sobre o impacto da covid-19 em pacientes com cardiopatias. Portanto, destacou-se o potencial risco do cardiopata e atuação do enfermeiro por meio de orientação em saúde por meio da telecomunicação (Pereira \& Correa, 2020).

Ávila \& Carvalho (2020) afirmaram que a triagem diferenciada melhorou o direcionamento das estratégias na assistência, tratamento e prevenção de complicações durante a gravidez e contribuiu significativamente para a redução dos 
efeitos adversos. Para os autores, enquanto não se estabelece uma estratégia de terapêutica específica, deve-se tratar e prevenir as complicações apresentadas por meio de cuidados integrados realizados pela equipe multidisciplinar para orientar e otimizar a terapêutica.

\section{Considerações Finais}

Observou-se que, apesar das pesquisas mostrarem que as gestantes possuem um maior risco para a infeção por covid19, alguns autores observaram que as consequências para o feto e recém-nascido também podem ser graves, principalmente se a infecção ocorrer no último trimestre da gestação. Em relação à assistência das gestantes, observou-se uma preocupação quanto a qualidade da assistência prestada, uma vez que muitas dessas gestantes necessitaram dos serviços públicos de saúde. Infelizmente, a carência de material científico que comprove as consequências da infecção pela covid-19 é um fato e isso impacta quanto à elaboração de protocolos de atuação para com as gestantes infectadas pela covid-19.

Diante desses obstáculos, faz-se imprescindível que o cuidado humanizado e individualizado se mantenha presente, pois as gestantes fazem parte de um grupo de pacientes que precisam de uma atenção crítica por conta de suas alterações fisiológicas e imunológicas. Além disso, a necessidade de proteger o feto representou uma maior responsabilidade com relação à prestação da assistência. Recomenda-se fortemente que os estudos acerca deste tema continuem com o objetivo de aprimorar a abordagem da equipe de enfermagem junto às gestantes cardiopatas acometidas pela covid-19.

\section{Referências}

Albuquerque, L. P., Monte, A. V. L. \& Araújo, R. M. S. (2020). Implicações da covid-19 para pacientes gestantes. Revista Eletrônica Acervo Saúde. 12(10). e4632. http://dx.doi.org/10.25248/reas.e4632.

Almeida, M. O., Portugal, T. M. \& Assis, T. J. C. F. (2020). Gestantes e covid-19: isolamento como fator de impacto físico e psíquico. Revista Brasileira Saúde Materno Infantil. 20(2). 603-606. http://dx.doi.org/10.1590/1806-93042020000200015.

Ávila, W. S. \& Carvalho, R. C. (2020). Covid-19: Um Novo Desafio para a Cardiopatia na Gravidez. Arquivos Brasileira de Cardiologia. 2020. 115(1). 1-4. http://dx.doi.org/10.36660/abc.20200511.

Cardoso, M. E. V et al. (2020) Covid-19 na gestação: uma revisão integrativa. Revista Eletrônica Acervo Saúde. 12(10). e4651. http://dx.doi.org/10.25248/reas.e4651.2020.

Cesário, J. M. S et al. (2021a). Assistência de enfermagem ao paciente oncológico submetido à anestesia. Research, Society and Development. 10(5). e31310514798. http://dx.doi.org/10.33448/rsd-v10i5.14798

Cesário, J. M. S. et al. (2021b). Cuidados de enfermagem para o paciente com infarto agudo do miocárdio portador de covid-19. Revista Científica Multidisciplinar Núcleo do Conhecimento. 2021. 12(2). 61-76. http://dx.doi.org/10.32749/nucleodoconhecimento.com.br/saude/infarto-agudo.

Cesário, J. M. S.; Flauzino, V. H. P \& Mejia, J. V. C. (2020) Metodologia científica: Principais tipos de pesquisas e suas caraterísticas. Revista Científica Multidisciplinar Núcleo do Conhecimento. 5(11). 23-33. http://dx.doi.org/10.32749/nucleodoconhecimento.com.br/educacao/tipos-de-pesquisas.

Estrela, F. M. et al. (2020). Gestantes no contexto da pandemia da Covid-19: reflexões e desafios. Revista de Saúde Coletiva. 30(2). 1-5.

Fagundes, M. C. M. et al. (2020). Anseios das profissionais de enfermagem gestantes frente à pandemia de covid-19. Rev. Enfermagem em Foco. 11(2). 109113.

Feitosa, I. P et al. (2020). Covid-19 e o Coração. Revista Científica Hospital Santa Izabel. 4(2). 77-88.

Flauzino, V. H. P. F et al. (2021). Paciente cardiopata em tratamento de câncer: Implicações para o cuidado de enfermagem. Research, Society and Development, 10(6) e34310615357. http://dx.doi.org/10.33448/rsd-v10i6.15357

Fonseca, A. J et al. (2020). Eletrocardiograma em gestante nos tempos da covid-19. Rev. Diagnóstico e Tratamento. 2020. 4(25). 159-161.

Guedes, B. L. C. S et al. (2020). Aspectos gerais da covid-19 na saúde de gestantes e recém-nascidos: uma breve revisão. Research, Society and Development. 9(7). e897974969. http://dx.doi.org/10.33448/rsd-v9i7.4969

Holanda, L. S et al. (2020). Infecção por covid-19 em Gestante Cardiopata. Arquivos Brasileiros de Cardiologia. 115(5). 936-938. http://dx.doi.org/10.36660/abc.20200517

Jesus, C. V. F et al. (2020). Gestante com covid-19 submetida à cesariana por sofrimento fetal: Primeiro relato de caso descrito no Brasil. ACM Arquivos Catarinenses de Medicina. 49 (2). 1-5. 
Research, Society and Development, v. 10, n. 9, e29610918097, 2021

(CC BY 4.0) | ISSN 2525-3409 | DOI: http://dx.doi.org/10.33448/rsd-v10i9.18097

Lima, J. R. et al. (2021). Estratégias de educação em saúde às gestantes e puérperas no enfrentamento à pandemia de covid-19. Research, Society and Development. 10(3). e36610313501. http://dx.doi.org/10.33448/rsd-v10i3.13501

Marquardt, M. H.; Bertoldi, L. F. \& Carvalho, F. R. S. (2020). Assistência de Enfermagem a gestantes atendidas nos serviços de saúde em tempos de pandemia: covid-19. Unesc em Revista. 4(2). 1-10.

Matos, J. H. F et al. (2021). Intervenções relacionadas às complicações cardiovasculares em pessoas hospitalizadas pela covid-19: revisão de escopo. Rev. Brasileira de Enfermagem. 2021. (74). 1-7. http://dx.doi.org/10.1590/0034-7167-2020-0568.

Melo, R. S. \& Cruz, N. S. (2020). Gestação no cárcere: garantia aos direitos reprodutivos em tempos de pandemia da covid-19. Revista Unifeso - humanas e sociais. 6(6). 83-92.

Nascimento, P. V., Cardoso, M. S. L. \& Neves, A. C. C. (2020). Principais desfechos fatais em indivíduos cardiopatas acometidos por covid-19. Rev. Enfermagem em Foco. 2020. 11(2). 46 -51.

Pereira, F. A. C. \& Correa, D. M. S. (2020). Uso da tele consulta pelo enfermeiro a cardiopatas: uma reflexão durante pandemia por covid-19 no Brasil. Revista de Enfermagem em Foco. 11(2). 167-171.

Peters, M. D et al. (2015). Guidance for conducting systematic scoping reviews. International Journal of Evidence-Based Healthcare. 2015. 13(3). 141-146. http://dx.doi.org/10.1097/XEB.0000000000000050.

Ribeiro, A. M. N et al. (2021). Repercussões da covid-19 para as gestantes. Research, Society and Development. 10(1). e2710111290. http://dx.doi.org/10.33448/rsd-v10i1.11290

Rodrigues, C. M. B et al. (2020). covid-19: sistema renal e cardíaco. Revista ULAKES Journal of Medicine. 1(EE). 60-66.

Rondelli, G. P. H. et al. (2020). Assistência às gestantes e recém-nascidos no contexto da infecção covid-19: uma revisão sistemática. Revista Desafios. 7(3). 48-74. http://dx.doi.org/10.20873/uftsuple2020-8943

Santos, C. M et al. (2020). Posicionamento sobre covid-19 e Gravidez em Mulheres Cardiopatas - Departamento de Cardiologia da Mulher da Sociedade Brasileira de Cardiologia. 115(5): 975-986. http://dx.doi.org 10.36660/abc.20201063

Teixeira, J. G et al. (2021). Gestantes covid-19 positivo, trabalho de parto e risco de transmissão vertical: revisão sistemática. Saúde coletiva. 11(60). http://dx.doi.org/10.36489/saudecoletiva.2021v11i60p4654-4663. 